\title{
Seuils prosaïques (notes sur quelques faits de poésie)
}

\section{Luigi Magno}

\section{(2) OpenEdition}

\section{Journals}

\section{Édition électronique}

URL : http://journals.openedition.org/rief/1057

DOI : 10.4000/rief.1057

ISSN : 2240-7456

\section{Éditeur}

Seminario di filologia francese

\section{Référence électronique}

Luigi Magno, «Seuils prosaïques (notes sur quelques faits de poésie) », Revue italienne d'études françaises [En ligne], 5| 2015, mis en ligne le 15 décembre 2015, consulté le 30 avril 2019. URL : http:// journals.openedition.org/rief/1057 ; DOI : 10.4000/rief.1057

Ce document a été généré automatiquement le 30 avril 2019.

\section{(c) (i) (9)}

Les contenus de la RIEF sont mis à disposition selon les termes de la Licence Creative Commons Attribution - Pas d'Utilisation Commerciale - Pas de Modification 4.0 International. 


\title{
Seuils prosaïques (notes sur quelques faits de poésie)
}

\author{
Luigi Magno
}

\section{RÉFÉRENCE}

Francis Ponge, Le Carnet du Bois de pins [1947], dans La Rage de l'expression [1952], CEuvres complètes, sous la direction de Bernard Beugnot, t. I, Paris, Gallimard, « Bibliothèque de la Pléiade ", 1999, p. 377-411.

Denis Roche, Prose au-devant d'une femme, Paris, Fourbis, 1988, p. 13-18.

Nathalie Quintane, Chaussure, Paris, P.O.L., 1997, p. 84-86.

\section{NOTE DE L'ÉDITEUR}

Luigi Magno a traduit les extraits des proses de Francis Ponge et de Denis Roche, Michele Zaffarano a traduit les extraits de Nathalie Quintane

1 Que reste-t-il de la prose en poésie au-delà ou en deçà du poème en prose ? On pourrait répondre à cette question par: des proses. Et préciser: des proses, aux identités aspectuelles multiples, génériquement non conventionnelles, que les poétiques ambiantes ont du mal à saisir. Parler de proses dans un dossier concernant la poésie, et non pas de poésies en prose ou de proses poétiques, ne relève en rien d'une provocation, ni d'un quiproquo. Il faudrait simplement s'accorder sur la signification à donner au mot proses (et à son italique). Mais la question définitoire, loin de résoudre le problème élémentaire seulement en apparence - le corse. $\mathrm{Si}$, pour schématiser, on exclut toute narration de type roman (et autres fabulations dans le genre), une définition liminaire de ces proses ne peut se penser qu'en négatif, en indexant tout ce qui n'est pas roman, n'est pas poésie en vers, n'est pas poésie en prose proprement dite. Une évidence cependant demeure, voire un fait de poésie déclencheur de ces lignes : si prose il y a en poésie, il est tout aussi bien vrai que « il y a prose et prose $»^{1}$. 
2 Repenser la notion de prose face à la poésie convoque tout d'abord la question du vers car, depuis ses origines, la poésie c'est le vers. Si pendant le XVIII ${ }^{e}$ siècle l'esthétique questionne déjà les échanges entre la prose et la poésie, ce n'est qu'au siècle suivant qu'on voit apparaitre en France, sous la plume de Baudelaire, l'expression " poème en prose ». C'est seulement au XIX ${ }^{e}$ siècle, en effet, que, dans le cadre d'une réaction à un certain lyrisme, la prose s'installe factuellement dans la poésie, que la poésie se désolidarise enfin du vers.

3 Comme en témoigne n'importe quelle histoire de la poésie ou du poème en prose, ce geste, tout en décloisonnant momentanément le système des genres, ouvre des voies inexplorées. Au milieu des formes diverses que ces proses ont connues, apparaît clairement la permanence d'une charge critique (au moins depuis Baudelaire), qui a servi non seulement à repenser les formes du poétique, mais surtout ses logiques. Ne pourraiton relire, par exemple, certains poèmes du Spleen de Paris comme un traitement des contextes discursifs des journaux et de la presse, ou encore certaines proses de Rimbaud comme une tentative de réinvention touchant l'usage de la mythologie tout comme des discours sur l'actualité ? Par l'adoption des proses, il s'agit pour certains, au moins depuis le Spleen de Paris, de " désaffubler » (Ponge) la poésie, lui ôter cette aura qui lui confère ontologiquement un statut de discours autre. En frottant le poème à «l'universel reportage ", Baudelaire contribue à sortir la poésie des jeux d'encodages qui la rendent un genre constitué, voire à l'éloigner d'une langue stylistiquement et rhétoriquement sublimante et codée, pour la déplacer, en briser certains ressorts, et la rapprocher un peu plus d'une expérience de langage parmi d'autres. Evidemment, tout ceci n'est pas encore clairement formulé dans cette perspective chez Baudelaire, qui garde tout au long de son œuvre une idée traditionnelle de l'art, mais les germes y sont (tout comme ils circulent chez Rimbaud et foisonnent chez Lautréamont).

4 Si le poème en prose a rendu disjonctif le rapport de la poésie au vers, l'apparition de formes poétiques complexes a dévoyé ultérieurement la classification générique de ses créneaux traditionnels, en propageant en milieu poétique des «objets verbaux non identifiés $\rrbracket^{2}$ dont le statut et le fonctionnement sont souvent difficiles à saisir. Car depuis que la prose et la poésie se joignent, se combinent et s'informent mutuellement, leurs formes respectives se multiplient et alimentent des configurations spécifiques et comme post-génériques, qui appellent à une re-sémantisation de la notion de prose (et de poésie avec elle). Penser à nouveaux frais la question des proses en poésie, essayer une suite ou série de notes au contact de ces quelques faits de poésie, veut dire proposer un sujet sérieux de réflexion sur lequel s'effritent la plupart des définitions les plus paradigmatiques de la poésie.

Revenir ici sur ces écritures à l'identité générique douteuse et inclassable mais dites tout de même poétiques ou étiquetées comme de la poésie, permet d'accomplir au moins deux tâches. Premièrement, il s'agit d'apporter une contribution au débat en cours et à la compréhension de ce territoire aux marges poreuses, de cette nébuleuse foisonnante qui inspire autant de méfiance chez certains que de curiosité scientifique chez d'autres. Deuxièmement, par une lecture des textes, il s'agit de soulever des questions poétologiques, génériques et esthétiques, que la circulation de ces proses ne cesse d'alimenter au moins depuis la moitié du $\mathrm{XX}^{\mathrm{e}}$ siècle en repoussant les limites et décloisonnant les marges du poétique.

6 La réflexion sur ces proses connaît en France une résonance depuis au moins une vingtaine d'années, grâce notamment aux travaux de Jean-Marie Gleize sur la littéralité. 
Pour mieux appréhender ces "objets spécifiques $"^{3}$ (comme il les appelle en écho à Donald Judd), Gleize n'hésite pas, à la suite de Baudelaire, Flaubert ou Ponge, à reprendre et à manipuler des " poétologèmes » construits autour de la notion de prose tels prose en prose, prose particulière, prose très prose, prose particulière aplatie, regroupant sous leur égide une grande variété d'objets verbaux qu'en France on n'hésite pas à considérer comme de la poésie4. En Italie, au contraire, la critique se montre assez méfiante envers cette production. Parfois elle plaque sur ces produits des étiquettes génériques traditionnelles, comme si, pour appréhender le fonctionnement de ces écritures, une vision essentialiste de la poésie, qui permettrait de parler de ces objets en termes d'appartenance, de champ, ou de possession de traits qualitatifs, pouvait suffire. Parfois, plus radicalement, elle exclut ces écritures du champ littéraire, et ce premièrement à cause du poids qu'une certaine ligne esthétique imbue d'idéalisme exerce encore aujourd'hui, malgré l'émergence de nouvelles écritures de recherche - dont témoigne l'anthologie Prosa in prosa ${ }^{5}$.

7 Si dans les quelques lignes qui précédent s'entasse déjà un certain nombre de noms pour ce qu'on présente ici comme des proses, c'est que les étiquettes foisonnent. Prose, proses en prose, prose plus prose, prose très prose, objet verbaux non identifiés, objets littéraires non identifiables, dispositifs, tests, objets spécifiques, enquêtes : voici juste un petit échantillonnage de la terminologie courante. Pour nous, ici, ces noms se superposent. On a insisté, à partir du titre, sur la notion de prose car elle permet d'inscrire ou en tout cas de comprendre ces écritures dans une perspective historique. Ces proses ne surgissent pas du néant, ne sont pas le caprice d'écrivains ou de poètes en recherche de visibilité, mais sont des formes que la littérature et la poésie s'approprient ou englobent comme autant de possibles après l'apparition du poème en prose proprement dit.

On a privilégié ici trois noms, qui sont autant de moments et d'échantillons possibles parmi d'autres de ces proses ou faits de poésie, appartenant à trois générations différentes. D'abord Francis Ponge, dont l'œuvre poétique se compose exclusivement (à quelques exceptions près) de proses et de proses; puis Denis Roche, poète-non-poète lui aussi qui produit des textes à partir d'instances plastiques et non discursives; enfin Nathalie Quintane, écrivaine dont les proses et les proses, génériquement inclassables, circulent en milieu poétique, continuant singulièrement et idéalement le travail des Ponge, des Roche...

9 Ces écrivains marquent trois moments et/ou espaces, incarnent trois façons de travailler et trois générations. Ils sont, à bien des égards, trois voies spécifiques d'une même nébuleuse qui partage certaines directions et certains soubassements communs. Si chez Ponge le poème en prose tel que la tradition le lui a légué est encore agissant, reconnaissable, fonctionnant par moments, celui-ci connaît très vite et en même temps dans son œuvre une transformation radicale jusqu'à sa négation. Avec Denis Roche on assiste à une gamme très variable d'imbrications formelles produites à partir de suggestions non littéraires (comme l'art abstrait ou la photographie), voire à une production de formes inédites qui n'ont plus rien à voir avec la tradition du poème en prose mais qui sont autant d'éclairages aspectuels (c'est-à-dire pluriels et variables). L'œuvre de Nathalie Quintane permet enfin de mesurer combien il faut repenser le problème de la prose en poésie en dehors de toute visée formelle et générique, car l'abondance de formes expérimentales, inclassables, et d'autres écritures de recherche définies en vrac comme de la poésie sollicite les chercheurs à réinvestir sémantiquement 
et re-conceptualiser un certain nombre de notions, trop souvent considérées comme acquises, stables, délimitables.

Par sa diversité et sa trajectoire qui s'étend sur presque tout le $\mathrm{XX}^{\mathrm{e}}$ siècle, l'œuvre de Francis Ponge constitue un véritable seuil. Ponge conçoit l'écriture comme une créationcritique où le texte n'est jamais exempt d'une réflexion sur lui-même, sans pour autant qu'il se renferme dans une boucle autotélique. S'il livre presque systématiquement en actes ses points de vue esthétiques et ses opinions critiques, c'est-à-dire à même ses textes de création, il n'est pas rare qu'il se positionne aussi ailleurs sur certaines questions, comme en 1971, lors d'un entretien avec Anthony Rudolf, quand il s'exprime directement sur les rapports entre prose et poésie :

Bien sûr j'ai lu les Poèmes en prose de Baudelaire et les proses de Mallarmé dans Divagations : sont-ce des poèmes en prose ? Cette antinomie entre poésie et prose est un non-sens. [...] Le fait qu'il n'y a plus de règles fixes de prosodie, proésie, signifie qu'il est impossible de classer intelligemment des proses comme poèmes et d'autres non. Une des premières anthologies de poèmes en prose d'après-guerre s'achève, je pense, sur moi. [...] L'anthologie commençait avec Parny au XVIII ${ }^{\text {ème }}$ siècle. Ensuite venaient Aloysius Bertrand, Michaux, moi-même. Mais mes textes critiques, mes textes sur la peinture par exemple, sont tout aussi difficiles, souvent plus difficiles, à écrire que ceux considérés comme poétiques. Je ne fais pas de différence. Mes audaces et mes scrupules sont les mêmes, quelque genre que vous assignez au texte. Mon premier recueil, publié en 1926, s'intitulait Douze petits écrits et s'ouvre avec trois ou quatre po... choses que l'on peut considérer comme des poèmes, si cela vous plait ; ils ont des lignes [...]. Ensuite Le Parti pris des choses. Paulhan et moi avons décidé d'éradiquer tout ce qui sentait la prosodie pour donner au livre une sorte d'unité, même visuelle. J'ôtai tout ce qui avait l'apparence de poème. ${ }^{6}$

11 Ici Ponge non seulement récuse la définition de poème en prose en ce qui concerne son œuvre, mais pointe en même temps les limites des définitions génériques traditionnelles ( «il est impossible de classer intelligemment des proses comme poèmes et d'autres non »), au moins de celles - la majorité - qui se fondent ontologiquement sur la possession de traits qualitatifs. Il souligne tout aussi explicitement la nécessité de repenser ses textes ( Mes audaces et mes scrupules sont les mêmes, quelque genre que vous assignez au texte ») à l'aune d'autres étiquettes (« choses que l'on peut considérer comme des poèmes »; « lignes »), donc d'autres poétiques, quitte à les inventer au besoin.

Pour mieux comprendre ces mots de Ponge il faut avoir à l'esprit son œuvre, à la fois dans son parcours et dans sa totalité. Au moment des Douze petits écrits (1926) ou encore du Parti pris des choses (1942), Ponge pense le texte comme une «bombe ", une formulation aboutie, close, définitive, qui ne trahit en rien le travail préalable de son élaboration. Après Le Parti pris des choses cependant, il se montre comme insatisfait de cette formeconception de l'œuvre et s'emploie à l'essayer, à en tester les possibilités, voire les possibles, afin de la décloisonner pour mieux l'exploiter. Il ne va pas renoncer à écrire en effet, mais continue de le faire tout en étant sous la férule d'une « rage de l'expression ». C'est alors qu'il conçoit la forme " proématique » (ou " proêmatique »), ni prose ni poésie, ou encore prose et poésie ensemble dans une configuration inédite, où prime la volonté de jeter une lumière critique sur l'ensemble de l'œuvre produite, aussi bien à travers des poèmes (en prose) ambigus - qui, tout en gardant une forme poétique codée, affichent les 
marques d'un processus de déconstruction in fieri - que par la publication de fragments disparates, comme les pages d'un journal ou des passages d'une conversation.

Le Carnet $d u$ Bois de pins (1947) marque en ce sens une étape importante et impose davantage la logique de ce nouveau projet. À partir de ce Carnet Ponge affiche une volonté critique de plus en plus radicale, tout en offrant en théorie et en pratique une vision de l'œuvre comme objet provisoire, comme suite de moments témoignant d'un processus de création en construction permanente. On a choisi de reprendre ici quelques passages du Carnet du Bois de pins car ce texte inaugure explicitement un geste majeur de rupture, à savoir la publication des brouillons comme œuvre, donc une conception de l'œuvre comme tension vers. Les extraits ici proposés montrent en même temps comment Ponge s'exerce aussi bien dans la construction d'un texte par rectification et ajustements continuels, que dans la critique de ce qu'il fait au fur et à mesure qu'il le fait. D'une part on assiste à l'exercice d'une poésie encore partiellement versifiée, métrifiée, soumise au chant, ou encore d'une prose poétique, voire d'une poésie en prose ; d'autre part, entre lignes et vers, se mêle l'expression forte d'une insatisfaction/déception consubstantielle à cet exercice, le refus d'une pratique de la poésie comme application de recettes toutes prêtes ou arrangement euphorique d'une parole, donc la volonté de poursuivre l'écriture par d'autres moyens et dans d'autres voies.

14 À partir de 1947 et en passant par des stratégies aussi disparates telles qu'elles circulent dans La Crevette dans tous ses états (1948), Pour un Malherbe (1965), Le Savon (1967), La Fabrique du pré (1971), Comment une figue de paroles et pourquoi (1977), Ponge ne cessera d'exhiber, de soumettre à "prostitution ${ }^{7}$ ses ateliers et leur négativité en tant qu'œuvre. Et c'est en revendiquant cet inachèvement, ce travail d'approximation, dans ces textes qui sont les travaux des textes (ou des textes sur les textes publiés en tant que texte), qu'il va concevoir l'œuvre comme " moviment $»^{8}$, un néologisme s'opposant à tout monument, à toute forme close et définitive. Cette conception de l'œuvre ne va pas sans un certain nombre de choix et de conséquences qui sont autant de moments de renouveau du poétique. Sans pouvoir ici développer sur ce point, il faut d'abord souligner l'utilisation de formes notatives, notulaires, constatives, littérales (carnets, journal, notes...) qui permet à Ponge de faire saillir un certain réel (ou le monde, si l'on préfère) et de garder donc la puissance des possibles à travers des formes et des noms inédits tels que " proême ", « sapate ", " carnet », « objeu », « fabrique ", « nioque ", pour n'en citer que quelques-uns. Et ce contre toute force assertive des discours (voici une conséquence de ce choix) qu'une certaine modernité avait, avant lui, pratiquée et revendiquée. La littérature/poésie demande un renouveau de ses formes qui est tout aussi bien un renouveau de ses logiques : elle rentre ainsi dans une aire critique, voire éthique, car elle met le lecteur face aussi bien aux stratégies constructives qui informent les textes qu'à des expériences de pensée.

Les avant-gardes des années 1960-1970 n'ont pas manqué, en France, de continuer la réflexion ouverte et aggravée par Ponge sur la poésie. Ce n'est pas un hasard si Ponge luimême se rapprochera à un moment de Tel Quel, s'il publie sa Figue dans le premier numéro de la revue éponyme, et surtout s'il reconnaît Denis Roche comme l'un de ses disciples ou continuateurs9.

Denis Roche en effet, tout au long de son œuvre, va poursuivre, par d'autres moyens et en exploitant d'autres modalités, l'interrogation que pose encore aujourd'hui l'œuvre de Ponge dans la direction d'une récusation de la Poésie ou d'un travail « sur ces formes du discours narratif que l'on nomme encore, sans doute par des impotences de lecture, 
"poésie" $»^{10}$. Certes, Roche participe, lors de son adhésion à Tel Quel, à une ambiance théorique d'ensemble qui essaie de repenser toute la littérature à l'aide d'autres disciplines, mais il sera aussi l'un des rares membres du comité de rédaction qui saura poursuivre sa réflexion et sa création dans un créneau personnel spécifique, loin du terrorisme théorique groupal ou d'autres obligations d'un militantisme aveugle. Et Ponge sera pour lui un modèle aussi bien avant et après sa sortie de Tel Quel en 1972. C'est dans cette même année qu'il rend hommage à Ponge dans ses textes poétiques ${ }^{11}$, et c'est sur lui qu'il va revenir encore en 1983, par exemple, quand il livre "La fabrique d'assez près ", des pages d'un journal où, par le biais des arts du visible, il reconsidère le travail de Ponge entre désusage des mots, «opacité du fourmillant réel » et approche frontale de la langue (« sans sinuosités, sans vertige, sans emphase ») $)^{12}$.

17 Après Le Mécrit (1972), Roche tourne le dos à la poésie en déclarant son inadmissibilité («La poésie est inadmissible, d'ailleurs elle n'existe pas »). Il faut lire cette affirmationmanifeste d'une part, en ce qui concerne son inadmissibilité, comme la nécessité de ne plus poursuivre une idée reçue de la poésie, voire la Poésie telle qu'elle est traditionnellement admise, comprise, écrite, lue; d'autre part, pour ce qui est de son existence, elle est à venir. C'est ainsi que la poésie de Roche se construit dans le refus de la Poésie, et se pense en même temps comme un laboratoire foisonnant de réinventions formelles et un chantier critique. C'est ainsi que la poésie, après Le Mécrit, sera pour Roche dans un roman comme Louve basse (1978), dans un livre inclassable comme les Dépôts de savoir \& de technique (1981), dans un journal tel les Essais de littérature arrêtée (1981), dans une conversation avec un photographe (en l'occurrence La Photographie est interminable, 2007), voire dans les multiples interfaces entre écritures et contextes représentationnels du visible ${ }^{13}$.

18 On a retenu ici un passage de Prose au-devant d'une femme. Cette micro-narration, qui, dans sa littéralité, se passe de commentaire, montre combien les textes de Roche après 1972 baignent copieusement dans cette création-critique qui est au cœur des dispositifs pongiens. Pour preuve, la phrase qui clôt l'extrait : «Or que peut un romancier contre un personnage qui a des idées bien arrêtées en matière de romans, sinon mentir par omission, au moins, et ruser de toute sa syntaxe? $»^{14}$. Ce doute syntaxique travaille l'esprit de Roche déjà en 1965, à l'occasion d'un compte rendu écrit suite à la parution de Comme $^{15}$. Dans ce recueil à deux têtes (poésie/prose), Marcelin Pleynet se plaît à essayer des stratégies de dédoublement autour de «ce qui se présente apparemment comme de la poésie » et de "ce qui se présente apparemment comme de la prose ». Non sans évoquer Lautréamont (sous l'égide duquel travaille explicitement Pleynet) et sa "vérité pratique " en poésie, ou ce que Ponge appelle "le dispositif Maldoror/Poésie », Roche pointe l'importance de repenser la syntaxe (" notre syntaxe ») et affirme : « Il est risible de croire qu'on puisse démystifier la poésie, quand seuls sont à démystifier certains procédés trop largement utilisés aujourd'hui comme "recettes" poétiques ». Le problème qui est posé, ici comme là, pourrait être reformulé, à l'échelle de toute l'œuvre de Roche, comme suit: est-ce qu'un écrivain peut, à travers une large stratégie syntaxique, repenser le fonctionnement d'un genre, voire une certaine idée de la poésie et/ou de la littérature?

La notion de syntaxe peut s'entendre, ici comme là, dans un sens large, à savoir comme un problème d'agencement. Il ne s'agit pas seulement d'un problème purement linguistique qui se résoudrait dans un ordre phrastique par exemple, ou dans le respect d'une norme grammaticale, ou encore dans la construction d'une ligne diégétique ou représentationnelle car «la poésie n'est pas un problème de représentation » ${ }^{16}$. La 
syntaxe serait plutôt, à bien des égards, une question de montage ou d'agencement pluriel et variable, c'est-à-dire un mode de traitement ou de ré-articulation d'un matériau discursif souvent déjà énoncé. Pour Roche, il s'agit d'une part de retraiter des discours émanant par exemple de l'infra-ordinaire et qui se présentent sous la forme d'un journal (comme les Essais de littérature arrêtée) ; d'autre part, et presque à l'opposé, de seconder les codes et les discours ambiants (comme dans les textes qu'il appelle dépôts ou antéfixes) tout en faisant jaillir, à travers des configurations inédites, de matériaux connus, un savoir critique, une connaissance, voire solliciter un geste, faire surgir une action. Et c'est dans ce dernier créneau que se concrétisent aussi les tentatives de modifier l'écriture et en perturber la logique par l'insertion d'expériences visuelles, donc de décliner, par exemple, l'art abstrait et la non-versification, la photographie et la littérature, la photographie comme littérature.

Si Ponge teste les paroles et parle contre elles, si Roche, après lui et d'après lui, s'emploie à rendre poreuses les interfaces littéraires, Nathalie Quintane radicalise en quelque sorte ces positions et repense le poético-littéraire au milieu de toutes ces expériences occasionnées par nos pratiques de vie, qu'elles soient publiques ou privées.

21 L'extrait ici présent est tiré de Chaussure, le deuxième livre de Quintane, publié en 1994. Le choix d'installer un objet dès le titre et de consacrer le livre au surgissement de l'objet dans le texte suffit pour lire Chaussure dans la lignée dessinée par l'œuvre de Ponge. Mais l'objet chéri par Quintane est plus problématique. Il n'appartient pas forcément au monde muet des choses, c'est un objet familier qui entraîne avec lui des connotations contradictoires, relevant par exemple à la fois du bas (qui existe dans son contact avec le sol) et du prisé (en tant que marchandise). Le ton du livre est lui aussi insaisissable et éclectique, passant d'une remarque à une lapalissade, d'un développement historique à des notes éparses. Or, le choix de l'objet chaussure ainsi que d'un ton extrêmement décousu, relève d'une stratégie complexe qui déplace les enjeux du poétique.

Quintane replace au centre du dispositif poétique ce qui relève du quotidien, du banal, de ce qui ne surgit pas forcément à la conscience réflexive. Cette opération n'a pas pour but de plaider la réévaluation d'un objet anodin comme une chaussure (il ne s'agit donc pas de révéler la beauté cachée d'une chaussure), ni d'étaler l'expérience perceptive d'un je au contact avec le monde (en ce sens il ne s'agit pas non plus d'une "poésie de la présence »). Ou mieux, l'expérience physique, le contact corporel et la perception matérielle de l'objet servent ici comme point de départ pour installer le quotidien au centre du livre. Ce quotidien, se déclinant sous les formes du banal, de l'anodin, de l'idiotie, présente un triple enjeu, car il est pour Quintane (et les écrivains de sa génération) à la fois un sujet du discours, mais aussi un générateur de discours, voire le domaine où puiser des outils pratiques d'enquête.

Avec Quintane la littérature semble quitter, seulement apparemment, son fonctionnement esthétique. On peut mesurer cette remise à plat du discours littéraire déjà dans le vocabulaire et/ou dans les outils employés : si Ponge construit sa méthode sur un socle encore classique (ne serait-ce que pour mieux le défaire) et si Roche insiste tout au long de son œuvre sur la notion de "Beauté » (qui peut toutefois s'entendre chez lui comme synonyme d'idiotie $)^{17}$, Quintane brasse les discours du quotidien sans pour cela les promouvoir, les polir, les rendre autres que ce qu'ils sont. C'est ainsi que le logos, interrogé par Ponge à partir d'un travail étymologique, se déplace chez Quintane vers une question de circulation et d'usage. Dans ce cadre épistémologique, l'expérience 
occasionnée par un texte littéraire ou une poésie (voire un tableau, une sculpture, une installation) n'a aucune spécificité ontologique, et encore moins de précellence.

Le travail de Quintane s'opère dans une perspective immanente et non surplombante, c'est-à-dire sans a priori, sans "parti pris ", sans aucune démonstration à faire. Pour cerner cette écriture et en saisir le fonctionnement, il faut alors se doter d'outils et de concepts qui se montrent solidaires d'expériences de vie quotidienne (faire du sport, écouter de la musique.... ${ }^{18}$. C'est sur ce terrain que l'esthétique rejoint davantage le quotidien et que la littérature ou la poésie se conçoivent, pour le dire avec Wittgenstein, comme un travail sur les "jeux de langage", agissant premièrement dans et sur nos " formes de vie ». Ces jeux de langage ramènent encore cette réflexion à un problème syntaxique. Pierre Alferi s'est employé, dans Chercher une phrase, à « définir la littérature par l'inquiétude de la syntaxe ». S'il voit dans la poésie « un lieu critique dans l'invention des phrases", qui comporte donc une "crise syntaxique ", cette crise «peut avoir lieu, plus discrètement, dans la prose, c'est-à-dire dans une mise en rythme purement syntaxique $»^{19}$. Or, ces observations sont à lire dans un cadre où « l'entrelacement de ces traits syntaxique n'est plus lui-même de nature syntaxique. (Un texte est plus qu'une longue phrase ou une grande forme, car, dans l'agencement de ses phrases, son idiome se donne toujours comme capable de produire d'autres agencements et d'autres phrases.) $»^{20}$ . La syntaxe devient alors une question éminemment cognitive, voire éthique (comme chez Ponge), car « Avant toute interprétation, toute analyse (rhétorique, stylistique ou autre), la manière dont le sens advient, le genre de consistance qu'il prend touchent le corps et sa pensée $»^{21}$. Et ce dans un contexte où «Penser veut dire: chercher une phrase », et où « La phrase invente une expérience $»^{22}$.

Encore une question pour finir. Qu'en est-il de ces proses en Italie? Pourquoi les accompagner de traductions? C'est là un geste qui va vers une plus grande diffusion, peut-être une lisibilité accrue. On évoquait au début le volume Prosa in prosa et, implicitement, les écritures d'auteurs comme Andrea Inglese, Gherardo Bortolotti, Alessandro Broggi, Marco Giovenale, Andrea Raos, Michele Zaffarano, qui ont animé ce projet. Ce recueil est, à plus d'un titre, un territoire où s'instaure un véritable dialogue entre la France et l'Italie sur le terrain de ces proses. Un dialogue tout récent, qui surgit dans un milieu non sans polémiques ${ }^{23}$, mais dont on pouvait déjà savourer un début en 1965, dans ces mots d'Italo Calvino, écrits suite à son énième lecture de Ponge, et qu'il faudrait juste reformuler aujourd'hui en remplaçant la «nostra narrativa » et les « forme romanzesche » par « nostra poesia » et « forme poetiche»:

Non è stato tante volte detto che la storia della nostra narrativa passa anche attraverso le carte dei cronisti e dei viaggiatori, le epistole, le ambascerie, gli exempla dei predicatori e ogni altro genere di scrittura pratica? Questa nozione di prosa dovrebbe ormai essersi riscattata dalla accezione lirica, evocativa, puristica che fu propria della "prosa d'arte »[...]; da tempo sappiamo che la vera prosa italiana del nostro secolo è quella di quando Gadda spiega il risotto o la chirurgia o il cemento armato. Se approfondiamo questa idea di prosa come scrittura che si impasta per formarci una spiegazione delle cose, sarà lì che potremo inserirci nella problematica attuale dell'écriture con qualcosa da dire, sarà di lì che i gamberetti di Ponge non ci saranno alieni [...]: di lì certo più che dalla parte dell'esaurimento delle forme romanzesche. ${ }^{24}$ 
ANNEXES 
Francis Ponge, extraits de Le Carnet du Bois de pins 


\section{LE PLAISIR DES BOIS DE PINS}

$[\ldots]$

9 août 1940 - Le soir

Non!

Décidément, il faut que je revienne au plaisir du bois de pins.

De quoi est-il fait, ce plaisir ? - Principalement de ceci : le bois de pins est une pièce de la nature, faite d'arbres tous d'une espèce nettement définie ; pièce bien délimitée, généralement assez déserte, où l'on trouve abri contre le soleil, contre le vent, contre la visibilité ; mais abri non absolu, non par isolement. Non! C'est un abri relatif. Un abri non cachottier, un abri non mesquin, un abri noble.

C'est un endroit aussi (ceci est particulier aux bois de pins) où l'on évolue à l'aise, sans taillis, sans branchages à hauteur d'homme, où l'on peut s'étendre à sec, et sans mollesse, mais assez confortablement.

Chaque bois de pins est comme un sanatorium naturel, aussi un salon de musique... une chambre, une vaste cathédrale de méditation (une cathédrale sans chaire, par bonheur) ouverte à tous les vents, mais par tant de portes que c'est comme si elles étaient fermées. Car ils y hésitent.

Ô respectables colonnes, mâts séniles !

Colonnes âgées, temple de la caducité.

Rien de riant, mais quel confort salubre, quelle températion des éléments, quel salon de musique sobrement parfumé, sobrement adorné, bien fait pour la promenade sérieuse et la méditation.

Tout y est fait, sans excès, pour laisser l'homme à lui seul. La végétation, l'animation y sont reléguées dans les hauteurs. Rien pour distraire le regard. Tout pour l'endormir, par cette multiplication de colonnes semblables. Point d'anecdotes. Tout y décourage la curiosité. Mais tout cela presque sans le vouloir, et au milieu de la nature, sans séparation tranchée, sans volonté d'isolation, sans grands gestes, sans heurts.

Par-ci, par-là, un rocher solitaire aggrave encore le caractère de cette solitude, force au sérieux.

Ô sanatorium naturel, cathédrale heureusement sans chaire, salon de musique où elle est si \{discète \{douce et reléguée

dans les hauteurs (à la fois si sauvage et si délicate), salon de musique ou de méditation - lieu fait pour laisser l'homme seul au milieu de la nature, à ses pensées, à poursuivre une pensée...

... Pour te rendre ta politesse, pour imiter ta délicatesse, ton tact (instinctivement je suis ainsi) - je ne développerai à ton intérieur aucune pensée qui te soit étrangère, c'est sur toi que je méditerai :

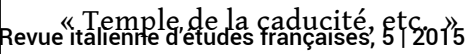


Il taccuino del bosco di pini 


\section{IL PIACERE DEI BOSCHI DI PINI}

$[\ldots]$

9 agosto 1940 - La sera.

No!

Bisogna decisamente che io torni al piacere del bosco di pini.

Di cosa è fatto, questo piacere ? - Principalmente di questo : il bosco di pini è una stanza della natura, fatta d'alberi tutti di una specie chiaramente definita ; stanza ben delimitata, generalmente piuttosto deserta, dove si trova riparo contro il sole, contro il vento, contro la visibilità ; ma riparo non assoluto, non per isolamento. No ! È un riparo relativo. Un riparo non segreto, un riparo non meschino, un riparo nobile.

È' anche un luogo (questo è peculiare ai boschi di pini) dove ci si muove con agio, senza legno ceduo, senza ramature a altezza d'uomo, dove ci si può sdraiare all'asciutto, e non sul morbido, ma abbastanza comodamente.

Ogni bosco di pini è come un sanatorio naturale, anche una sala di musica... una camera, una vasta cattedrale di meditazione (una cattedrale senza pulpito, per fortuna) aperta a tutti i venti, ma da così tante porte che è come se fossero chiuse. Infatti, essi vi restano esitanti.

O venerande colonne, senili alberi ! Colonne anziane, tempio della caducità.

Niente di ridente, ma che comodità salubre, che tempra degli elementi, che sala di musica sobriamente profumata, sobriamente adorna, adatta alla passeggiata seria e la meditazione.

Tutto è fatto, senza eccessi, per lasciare l'uomo da solo. La vegetazione, l'animazione sono relegate in alto. Nulla che distragga lo sguardo. Tutto per addormentarlo, attraverso questa moltiplicazione di colonne simili. Nessun aneddoto. Tutto scoraggia la curiosità. Ma tutto questo quasi senza volerlo, e in mezzo alla natura, senza separazione netta, senza volontà d'isolamento, senza grandi gesti, senza urti.

Qui e là, una roccia solitaria aggrava ancora il carattere di questa solitudine, costringe al serio.

O sanatorio naturale, cattedrale per fortuna senza pulpito, sala di musica dov'essa è così \{discreta

\{dolce e relegata

in alto (così selvaggia e così delicata insieme), sala di musica o di meditazione - luogo fatto per lasciare l'uomo solo in mezzo alla natura, ai suoi pensieri, a perseguire un pensiero...

... Per ricambiare la cortesia, per imitare la tua delicatezza, il tuo tatto (istintivamente sono così) - non svilupperò al tuo interno un pensiero che ti sia estraneo, è su di te che mediterò :

"Tempio della caducità, ecc."

\footnotetext{
"Credo che comincio a rendermi conto del piacere proprio dei boschi di pini."
Revue italienne d'études françaises, $5 \mid 2015$
}

$[\ldots]$ 
Denis Roche, extrait de Prose au-devant d'une femme 
$[\ldots]$

Quand le cheval et la femme ne furent plus qu'à quelques mètres de moi, l'inventaire devint possible. Le cheval, la robe du cheval ; la femme, la robe de la femme ; la couleur d'ensemble les couleurs des peaux et des étoffes ; l'allure de l'un, le maintien de l'autre.

Constatons le paradoxe du torse dressé de la femme, reins cambrés, visage levé, et du bassin dilaté, encombrant, cuisses écartées : magique échafaudage charnel dans un contenant de rêve et dont le fond tape en cadence à travers un film de sueur sur l'échine raide d'un cheval au regard mol. Trop de mots mis ainsi ensemble trahissant la lourdeur, l'effroi de ce moment d'écriture, car il se peut, je pense qu'il se peut, que des circonstances entachées de vulgarité (et on ne me dira pas que la vision du ventre de Blanche Castle affaissé sur le dos de ce cheval, tout nuageusement que je pouvais imaginer ses entrailles roses, ou tout fébrilement son pubis comme du ciré noir plongeant dessous, ou tout bêtement son nombril oscillant au rythme de l'avancée du cheval, n'était pas terriblement entachée de vulgarité, n'appelait pas un trop plein de la phrase) ne puissent se contenter de phrases comme toutes les autres, bien filées, d'une prose comme il faut où l'auteur dirait, la plume en l'air : " Comme cette apparition était vulgaire! ", etc. Le miroir, promené le long des chemins, n'en voit pas que de belles.

À l'instant où le cheval s'arrêta à ma hauteur et où, du coup, le léger balancement qui animait Blanche Castle cessa, j'étais pris par le souvenir très loin mais très net d'un moment presque similaire par son obscénité, mais cette autre fois c'était une obscénité admirable qu'avait fixée sans le savoir un photographe laborieux mais efficace qui m'avait confié, à des fins d'édition, un gros album rempli de photos de contorsionnistes. Il s'agissait, m'avait-il dit, de " photos de travail " destinées à mettre en valeur, non pas ses talents de photographe, mais la virtuosité, la singularité, le savoir faire extraordinaires de ces très jeunes femmes posant quasiment nues dans des poses qu'un reptile adolescent n'aurait pu prendre, et affairées dans une immobilité morbide de corps disjoint, noué en huit, avec des parties comme extravasées, d'autres, qu'on ne voit généralement pas, projetées en gros plan, avec ça et là un visage si joli, très souriant, surgissant face à vous entre des cuisses courbées comme un arc qui va casser. Le décor de ces images n'était pas le moins indécent de l'exhibition, car ces femmes vivant en général dans des caravanes, chacune avait posé à l'endroit qui avait paru le plus apte à mettre en valeur sa prestation, c'est-à-dire juchée sur la table de formica dans le coin cuisine de l'habitacle.

Plus tard, un soir où Blanche Castle, allongée contre moi sous les arbres, murmurait à ma demande des souvenirs troubles, je faillis lui parler de l'album des contorsionnistes, mais je doutais alors que l'association de cette imagerie anatomiquement rocambolesque avec sa première apparition à cheval dans la cour de l'abbaye pût avoir la moindre chance de lui plaire. Blanche, qui n'avait guère de principes, croyait dur comme fer à la vérité des romans et de leurs clichés, et notamment qu'une héroïne qui apparaît est l'occasion d'un morceau de bravoure où le narrateur doit faire preuve de sa maestria à décrire le visage qu'il va aimer, et suffisamment en détail pour que le lecteur croie que ce visage est vrai et qu'il est si beau que l'amour que va lui porter notre homme en sera d'autant plus plausible. Avouer à Blanche qu'en la voyant pour la première fois, je n'avais eu d'yeux que pour l'affaissement de son ventre sur l'échine raide de son cheval et que ma première pensée avait été pour un album de photos de cirque qu'apparemment la censure qui régnait à l'époque avait empêché tout le monde de publier, aurait eu quelque chose d'un blasphème envers sa beauté, bien évidemment, et d'une insulte envers les bonnes manières romanesques. Or que peut un romancier contre un personnage qui a des idées bien arrêtées en matière de romans, sinon mentir par omission, au moins, et ruser de toute sa syntaxe?

$[\ldots]$ 
Prosa al cospetto di una donna 
$[\ldots]$

Quando il cavallo e la donna non furono più che a pochi metri da me, l'inventario divenne possibile. Il cavallo, il mantello del cavallo ; la donna, il mantello della donna ; il colore d'insieme i colori delle pelli e delle stoffe, l'andatura dell'uno, il contegno dell'altra.

Notiamo il paradosso del busto eretto della donna, reni inarcate, testa alta, e del bacino dilatato, ingombrante, cosce divaricate : magica impalcatura carnale in un contenente da sogno e la cui base batte in cadenza attraverso una pellicola di sudore sul dorso rigido di un cavallo dallo sguardo molle. Troppe parole messe così insieme a tradire la pesantezza, lo sforzo di questo momento di scrittura, perché è possibile, credo sia possibile, che delle circostanze macchiate di volgarità (e non mi si dirà che la visione del ventre di Blanche Castle curvo sulla schiena di questo cavallo, per quanto nebulosamente potessi immaginare le sue viscere rosa, o febbrilmente il suo pube come un'incerata nera) non possano accontentarsi di frasi come tutte le altre, ben costruite, di una prosa come si deve dove l'autore direbbe, con la penna in aria : “Quanto era volgare questa apparizione !", ecc. Lo specchio, che passa per le vie, non ne vede solo delle belle.

Nell'istante in cui il cavallo si fermò alla mia altezza e in cui, improvvisamente, il lieve dondolio che animava Blanche Castle cessò, ero preso dal ricordo lontanissimo ma molto preciso di un momento quasi simile nella sua oscenità, ma quella volta si trattava di un'oscenità ammirevole che aveva fissato senza saperlo un fotografo vanamente operoso ma efficace che mi aveva affidato, per scopi editoriali, un grande album pieno di foto di contorsioniste. Si trattava, mi aveva detto, di "foto di lavoro" destinate a mettere in risalto, non il suo talento fotografico, ma la virtuosità, la singolarità, le capacità straordinarie di quelle giovani donne che posavano quasi nude in pose che nemmeno un rettile adolescente avrebbe potuto assumere, indaffarate nella morbosa immobilità di un corpo sconnesso, annodato a forma di otto, con parti come stravasate, altre, che di norma non si vedono, proiettate in primo piano, con qua e là un così bel viso, molto sorridente, che emerge di fronte a voi tra cosce curve come un arco che sta per spezzarsi. Lo sfondo di quelle immagini non era la cosa meno indecente dell'esibizione, poiché, vivendo spesso quelle donne in una roulotte, ciascuna aveva posato nel posto che era parso più adatto a mettere in risalto la propria presentazione, cioè appollaiata sul tavolo in formica nell'angolo cottura dell'abitacolo.

Qualche tempo dopo, una sera in cui Blanche Castle, sdraiata addosso a me sotto gli alberi, sussurrava su mia sollecitazione torbidi ricordi, poco mancò che non le parlassi dell'album delle contorsioniste, ma dubitavo allora che l'associazione tra quella iconografia anatomicamente rocambolesca e la prima apparizione di lei a cavallo nel cortile dell'abbazia potesse avere anche solo una minima possibilità di piacerle. Blanche, che non aveva molti princìi, credeva ciecamente nella verità dei romanzi e dei loro clichés, e segnatamente che un'eroina che entra in scena rappresenta un'occasione per un pezzo di bravura in cui il narratore deve far prova di maestria nel descrivere il volto che amerà, sufficientemente in dettaglio perché il lettore creda che questo viso sia vero e così bello da rendere tanto più plausibile l'amore che manifesterà per esso il nostro uomo. Confessare a Blanche che vedendola per la prima volta, non avevo avuto occhi che per lo sprofondare del suo ventre sulla schiena rigida del cavallo e che il mio primo pensiero era andato a un album di fotografie circensi che, a quanto pare, la censura regnante all'epoca aveva impedito a tutti di pubblicare, avrebbe avuto, era ovvio, qualcosa di blasfemo nei confronti della sua bellezza, e di ingiurioso nei confronti delle buone maniere romanzesche. Ora, cosa può un romanziere contro un personaggio che ha idee irremovibili sul romanzo, se non mentire almeno per omissione e agire con astuzia sulla sintassi?

$[\ldots]$ 


\title{
Nathalie Quintane, extrait de Chaussure
}

\author{
$[\ldots]$
}

KROUTCHEV

Kroutchev était alors assis à une table. Il avait pour ainsi dire l'allure d'un homme important, son air. Aussi était-il, en effet, un homme important. Mais qu'est-ce que cet air ? Une lueur (un éclat) dans l'œil (dans le regard) ? Deux épaules impassibles ? L'air : d'être debout même quand on est assis ? Le fameux claquement produit par la fermeture d'un dossier ? Les mains, posées à plat, sur ce dossier fermé ? L'air : d'être de dos quand on est de face ? Ce dos, lui-même expressif ? L'expressivité de ce dos qui parle, muet? L'air broussailleux? Bref, à ce moment précis, Kroutchev avait l'air. Or, soudain, Kroutchev avait mis sa chaussure sur la table, et tapé à plusieurs reprises avec celle-ci (toujours sur la table), si bien qu'on ne vit plus qu'elle. Soudain, sa chaussure avait remplacé Kroutchev : une chaussure de ville, montante, presque un godillot, sans caractéristiques particulières, bref, le type même du soulier banal, la plus ordinaire des chaussures. Or, Kroutchev tapait du pied, cette chaussure à la main, ou plutôt, la chaussure de Kroutchev tapait du pied pour lui. Si bien que Kroutchev disparaissait, momentanément, derrière.

Non qu'il ait effectivement disparu, la chaussure ayant pris, par son élévation à table, des proportions hors normes, mais, aux yeux du spectateur, son air, lui, avait bel et bien disparu, brouillé par le surgissement impromptu d'un objet certes banal, mais déplacé. La chaussure était, de toute façon, bien plus grosse que le seul nez de Kroutchev ; cela, il fallait l'accepter. Elle était même pratiquement de la hauteur de son visage, en tout cas, placée à sa hauteur, on voyait bien qu'elle s'y encadrait, et que celui-ci la dépassait à peine - éclipse partielle du visage de Kroutchev.

Les chaussures d'Imelda Marcos prennent la place d'un train, d'un paquebot, d'un avion supersonique. En rentrant au pays, Imelda Marcos demanda : où étaient ses chaussures ? Où avaient-elles bien pu passer? Qu'en avez-vous fait? Que sont-elles devenues?

Tous les matins, Imelda Marcos s'entretient avec elle-même - Laquelle porter d'entre toutes?

Tous les soirs, Imelda Marcos expose ses chaussures aux rayons du soleil couchant - qu'elles aient le pouvoir de m'emmener jusqu'au bout du monde.

Certaines chaussures d'Imelda Marcos conviennent très bien comme chapeau.

Imelda Marcos change de chaussures quand elle sort de table.

C'est de la graisse de chat qu'on fabrique pour les cirer.

Imelda Marcos s'endort avec des chaussons de lisière. Parfois, pendant la nuit, un chausson se perd au fond de son lit. Elle se retrouve un pied nu le matin.

Lorsqu'une chaussure tombe sur le côté quand elle la quitte, elle la redresse aussitôt.

Imelda Marcos pleura quand elle perdit ses escarpins mauves.

Imelda Marcos a une collection de chausse-pieds en corne, en acajou, en lapis-lazuli, en ivoire et en ébène.

$[\ldots]$ 


\section{Scarpa}

$[\ldots]$

\section{KRUSCIOV}

In quel momento, Krusciov se ne stava seduto a un tavolo. Aveva per così dire il portamento, l'aria di una persona importante. $\mathrm{E}$, in effetti, era una persona importante. Ma cos'è quest'aria ? Una luce (un lampo) negli occhi (nello sguardo) ? Due spalle impassibili ? L'aria : di stare in piedi anche quando si sta seduti ? Il famoso colpo prodotto dalla chiusura di un dossier ? Le mani appoggiate distese su questo dossier chiuso ? L'aria : di stare di schiena anche quando si sta di fronte ? La schiena, anche lei espressiva ? L'espressività di questa schiena che parla, muta ? L'aria arruffata ? Insomma, in quel preciso istante, Krusciov aveva l'aria.Ecco che, all'improvviso, Krusciov aveva messo la scarpa sul tavolo e, con quella, battuto a più riprese (sempre sul tavolo), tanto che a un certo punto si vedeva solo lei.

All'improvviso, la scarpa aveva sostituito Krusciov : una scarpa da città, alta, quasi uno scarponcino, senza particolari caratteristiche, insomma, l'esempio tipico di scarpa banale, la più ordinaria delle scarpe. Ecco, con questa scarpa in mano, Krusciov batteva il piede o meglio, la scarpa di Krusciov batteva il piede per lui. Tanto che, per il momento, Krusciov ci scompariva dietro.

Non che fosse veramente scomparso per colpa delle proporzioni fuori norma assunte dalla scarpa sollevata sul tavolo, però, agli occhi dello spettatore, la sua aria, quella sì, era davvero scomparsa, scompigliata dal sorgere improvviso di un oggetto sicuramente banale ma fuori posto. Ad ogni modo, la scarpa era persino più grossa del naso di Krusciov da solo ; e questo era un fatto che andava accettato. In pratica, era della stessa altezza del viso di lui, piazzata in ogni caso alla sua stessa altezza, e si vedeva bene che rientrava dentro il suo quadro, che questo quadro la sorpassava appena - eclisse parziale del volto di Krusciov.

Le scarpe di Imelda Marcos prendono il posto di un treno, di un traghetto, di un aereo supersonico.

Rientrando in patria, Imelda Marcos ha chiesto : dov'erano le sue scarpe ? Dove potevano essere finite? Che cosa ne avete fatto? Che cosa gli è successo?

Tutte le mattine, Imelda Marcos s'intrattiene con sé stessa - Fra tutte, quale portare?

Tutte le sere, Imelda Marcos espone le proprie scarpe ai raggi del sole al tramonto - che abbiano il potere di portarmi ai confini del mondo.

Alcune scarpe di Imelda Marcos potrebbero andare benissimo come cappello.

Imelda Marcos cambia scarpe quando si alza da tavola.

Per lucidarle, viene prodotto grasso di gatto.

Imelda Marcos va a dormire con delle pantofole di stoffa. A volte, durante la notte, una pantofola si perde in fondo al letto. La mattina, lei si ritrova un piede nudo.

Se lasciando una scarpa questa cade sul fianco, lei la rimette subito in piedi.

Imelda Marcos ha pianto quando ha perso le sue scarpette malva.

Imelda Marcos ha una collezione di calzascarpe di corno, di mogano, di lapislazzuli, d'avorio e di ebano.

$[\ldots]$ 


\section{NOTES}

1. Cette citation reprend le sous-titre de L'Hexaméron, un roman collectif publié en 1990 aux éditions du Seuil. Dans la quatrième de couverture les auteurs précisent : « Quant au sous-titre du livre, "Il y a prose et prose", libre à chacun de l'entendre comme il voudra, selon son humeur ou sa curiosité : c'est peut-être un simple constat, ou une provocation ». Ces lignes se présentent littéralement comme une affirmation apparemment assez banale au sujet de la pluralité des proses nourrissant le livre en question - où chaque chapitre est écrit par un auteur différent -, et à partir desquelles on pourrait par exemple développer une analyse théorique qui irait quelque part dans la direction d'une nouvelle «poétique de la prose » ou une " grammaire du récit » (cf. T. Todorov, Poétique de la prose, Paris, Seuil, 1971 et 1978). Or, la présence, au sein du collectif, de poètes tels Michel Deguy, Denis Roche et Jacques Roubaud, autorise à reconsidérer ce constat, comme on souhaite le faire ici, à l'égard de la poésie (cf. L'Hexaméron, Paris, Seuil, 1990).

2. P. Alferi, O. Cadiot, «La mécanique lyrique », dans Revue de littérature générale, 1, 1995, p. 3-22, aux p. 5-7.

3. J.-M. Gleize, « Vers des objets spécifiques », dans Europe, 755, 1992, p. 20-31.

4. Cf. J.-M. Gleize, A noir. Poésie et littéralité, Paris, Seuil, 1992 (repris dans J.-M. Gleize, Littéralité, Paris, Questions théoriques, 2015, p. 337-540) ; Id., Sorties, Paris, Questions théoriques, 2009 et 2014.

5. Sur ce débat en Italie cf. P. Giovannetti, Dalla poesia in prosa al rap. Tradizioni e canoni metrici nella poesia italiana contemporanea, Novara, Interlinea, 2008 ; Id., « Dopo il sogno del ritmo. Installazioni prosastiche della poesia », dans Prosa in prosa, Firenze, Le Lettere, 2009, p. 5-17. Pour un regard croisé sur la France et l'Italie cf. A. Inglese, « Poesia in prosa e arti poetiche. Una ricognizione in terra di Francia », 31 mars 2010, http://www.nazioneindiana.com/2010/03/31/poesia-in-prosa-earti-poetiche-una-ricognizione-in-terra-di-francia/ et 6 avril 2010, http:// www.nazioneindiana.com/2010/04/06/poesia-in-prosa-una-ricognizione-in-terra-di-francia-2/.

6. F. Ponge, « Prose ou poésie », in Id., Euvres complètes, tome II, sous la direction de B. Beugnot, Paris, Gallimard, « Bibliothèque de la Pléiade », 2002, p. 1409.

7. F. Ponge, La Fabrique du pré, Paris, Skira, 1971, Prière d'insérer.

8. F. Ponge, Écrit Beaubourg, in Id., Euvres complètes, tome II, sous la direction de B. Beugnot, Paris, Gallimard, « Bibliothèque de la Pléiade », 2002, p. 895-910.

9. Cf. F. Ponge, «Voici déjà quelques hâtifs croquis pour un "portrait complet" de Denis Roche », dans TXT, 6/7, 1974, p. 19-22. Sur Ponge et Roche cf. J.-M. Gleize, A noir, Paris, Seuil, 1992, p. 175-189 ; S. Baquey, «Francis Ponge, Denis Roche et le projet moderne en poésie ", dans Ponge, résolument, Lyon, ENS éditions, 2004, p. 165-178.

10. D. Roche, Éros énergumène, Paris, Seuil, 1968, quatrième de couverture.

11. Cf. D. Roche, «Les tentations de Francis Ponge ", dans Id., Le Mécrit, Paris, Seuil, 1995, p. 579-586.

12. D. Roche, «La fabrique d'assez près », dans Id., Dans la maison du Sphinx, Paris, Seuil, 1992, p. 133-148, aux p. 136-137 et 143-144.

13. On pourrait appliquer à Denis Roche lui-même ce qu'il écrit à propos de Ponge: "Ponge n'évolue pas [...]. Il construit comme le puzzle d'un arbre (on sait sa prédilection pour l'image de l'arbre littéraire), chaque morceau du puzzle étayant, dessinant peu à peu l'ensemble, comblant vide après vide, (là encore, Lucrèce), pliant ses mots à l'injonction de l'ensemble figuré comme éventuel (en cours de travail) et définitif (vers sa fin, comment savoir quelle "dernière touche" sera la dernière du tableau, imprévisible moment décisif) » (D. Roche, "La fabrique d'assez près ", dans Id., Dans la maison du Sphinx, Paris, cit., p. 133-148, à la p. 146).

14. D. Roche, Prose au-devant d'une femme, Paris, Fourbis, 1988, p. 17-18. 
15. D. Roche, «La pratique de la poésie », dans Faire Part, 30-31, p. 91-92.

16. D. Roche, Récits complets, Paris, Seuil, 1963, p. 15.

17. À ce sujet cf. J. Sivan, Le beau en présence ou le sexe à la mère Denis, Paris, Java, 1992.

18. Sur cette question cf. J.-M. Schaeffer, L'expérience esthétique, Paris, Gallimard, 2015.

19. P. Alferi, Chercher une phrase, Paris, Christian Bourgeois, 1991, p. 26.

20. Ibid., p. 68.

21. Ibid., p. VIII.

22. Ibid., p. 45 et p. 41.

23. On pense ici aux échanges entre Alfonso Berardinelli d'une part, Andrea Inglese et Andrea Raos d'autre part, déclenchés lors de la publication d'un article de ces derniers présentant pour la première fois en Italie - et par un geste militant, qui plus est - des écrivains comme Nathalie Quintane, Christophe Tarkos, Olivier Cadiot, Pierre Alferi.

24. I. Calvino, "Notizia su Giorgio Manganelli », dans Id., Saggi. 1945-1985, a cura di M. Barenghi, Milano, Mondadori, 1995, pp. 1153-1158, à la p. 1156 [déjà dans : Il menabò di letteratura, 8, 1965, pp. 102-105]. « N'a-t-on pas souvent dit que l'histoire de nos récits en prose passe aussi par les papiers des chroniqueurs et des voyageurs, les épîtres, les correspondances diplomatiques, les exempla des prêcheurs et tout autre type d'écriture pratique? Cette notion de prose devrait désormais s'être rachetée de l'acception lyrique, évocatoire, puriste qui était typique de la "prose d'art" [...]; depuis longtemps nous savons que la véritable prose italienne de notre siècle demeure dans les pages où Gadda explique le risotto ou la chirurgie ou le béton armé. Si nous explorons cette idée de prose comme d'une écriture qui se pétrit pour nous former une explication des choses, c'est là que nous pourrons intervenir dans la problématique actuelle de l' écriture avec quelque chose à dire, c'est à partir de là que les crevettes de Ponge ne nous seront pas étrangères [...] : de là plus que du côté de l'exténuation des formes du roman » (c'est moi qui traduis).

\section{RÉSUMÉS}

Cet essai propose une réflexion sur certaines écritures contemporaines dites poétiques et qui, tout en adoptant formellement de la prose, ne relèvent pas de catégories génériques couramment admises, tel le poème en prose (proprement dit) ou la prose poétique. À partir de l'œuvre de Francis Ponge, de Denis Roche et de Nathalie Quintane, cet article formule quelques clés de lecture de ces écritures "trans-génériques » ou "post-génériques » et se veut, en dernière analyse, une contribution au débat poétologique ambiant sur ce sujet. Il s'agit de repenser le fonctionnement de ces objets verbaux à partir de définitions non qualitatives, qui prennent en compte le texte et « l'acte » du texte comme autant de traits consubstantiels à l'œuvre.

\section{INDEX}

Mots-clés : prose, prose en prose, poème en prose, poétique, création-critique, Ponge (Francis), Roche (Denis), Quintane (Nathalie), Gleize (Jean-Marie) 